\title{
Delivery of Salbutamol Pressurized Metered-Dose Inhaler Administered Via Small-Volume Spacer Devices in Intubated, Spontaneously Breathing Rabbits
}

\author{
JEAN-CHRISTOPHE DUBUS, RODNEY RHEM, SHELLEY MONKMAN, AND MYRNA DOLOVICH
}

Department of Pediatrics, CHU Timone-Enfants, Marseille, France [J.-C.D.]; Departments of Medicine [R.R., M.D.] and Pediatrics [S.M.], McMaster University Medical Centre, Hamilton, Ontario, Canada.

\begin{abstract}
Little is known about the ability of small-volume valved spacer devices to deliver a significant amount of an aerosolized drug to the lungs of babies. This study compared the in vitro delivery of salbutamol administered via Aerochamber-Infant $(145 \mathrm{~mL})$, Babyhaler $(350 \mathrm{~mL})$, and metallic NES-spacer $(250$ $\mathrm{mL}$ ), as well as the in vivo delivery using an animal model. The lung deposition study of technetium-99m-labeled salbutamol was conducted in six anesthetized, intubated (3.0-mm endotracheal tube simulating oropharyngeal deposition), spontaneously breathing New Zealand White rabbits, a model for 3-kg babies. Each rabbit was studied on three separate occasions, once with each spacer device. The amount of radioactivity deposited in the spacer device, the endotracheal tube, the lungs, or the body was measured by a gamma camera and expressed as a percentage of the emitted labeled dose. The emitted dose and particle size distribution of salbutamol via the three spacer devices were
\end{abstract}

\section{ABSTRACT}

measured using unit dose sampling tubes and an eight-stage Anderson cascade impactor, respectively. The results were compared by ANOVA or Student-Newman-Keuls test when indicated. In vitro, the NES-spacer and Babyhaler were equivalent for delivering particles $<5.8 \mu \mathrm{m}$ in diameter (NES-spacer $=$ Babyhaler $>$ Aerochamber-Infant; $p<0.05$ ). In vivo, the lung and body deposition was low with all spacer devices (range: $0.52-5.40 \%$ of the delivered dose) but greater with the NESspacer than with the Aerochamber-Infant or the Babyhaler (5.40 $\pm 2.40 \%, 2.91 \pm 0.86 \%, 0.52 \pm 0.46 \%$, respectively; $p=$ 0.002 ). These results suggest the metal-valved spacer device may be preferable for delivering pressurized aerosols to spontaneously breathing infants. (Pediatr Res 50: 384-389, 2001)

\section{Abbreviation \\ BDP, bronchopulmonary dysplasia}

Small-volume spacer devices fitted with a face mask and inspiratory/expiratory valves have been developed for delivering pressurized metered-dose inhaler drugs to infants and newborns with various respiratory diseases (1-4). However, little is known about the ability of these spacer devices to deliver a significant amount of drug to the lungs of spontaneous breathing infants and a fortiori in intubated and mechanically ventilated infants and newborns.

The radiotracer technique is the only method available for direct estimation of aerosol deposition in vivo. This technique is commonly used in adults, and has been used in several studies in infants (5-7). However, the exposure of small infants to radioisotopes remains a concern. Thus, performance is often projected from in vitro experiments $(8-10)$ or in vitro lung models $(11,12)$, with data obtained at lower inspiratory flow

Received January 12, 2000; accepted March 6, 2001.

Correspondence: Jean-Christophe Dubus, M.D., Ph.D., Service de Médecine Infantile, CHU Timone-Enfants, 13385 Marseille Cedex 5, France; e-mail: jdubus@ap-hm.fr

Supported, in part, by a grant from Glaxo-Wellcome Laboratories (Paris, France) and from Astra Draco Laboratories (Nanterre, France). rates and higher tidal volumes through the spacer devices than what would be encountered in clinical practice. Animal "infant" models $(13,14)$ may be suitable alternatives to assess pulmonary deposition of labeled drugs. Usually, rabbits are tracheotomized, mechanically ventilated, and killed, but this animal model poorly reflects the lung deposition of drug administered through spacer devices in spontaneously breathing children. Because oropharyngeal deposition is low when using spacer devices and comparable to that deposited in an endotracheal tube (7), a better approach may be to use intubated, spontaneously breathing rabbits, not ventilated at the time of aerosol administration, to mimic spontaneously breathing infants treated with pressurized metered-dose inhalers and spacer devices.

The purpose of the study was two-fold: first, to compare the in vitro characteristics of salbutamol aerosol delivered from a pressurized metered-dose inhaler via three small-volume valved spacer devices [i.e. plastic Babyhaler, $350 \mathrm{~mL}$ (GlaxoSmithKline, Uxbridge, Middlesex, U.K.); plastic Aerochamber-Infant, $145 \mathrm{~mL}$ (Trudell Medical International, London, 
Ontario, Canada); and metallic NES-spacer, also called Nebuchamber, $250 \mathrm{~mL}$ (Astra Draco, Lund, Sweden)] used to deliver pressurized metered-dose inhaler aerosols to infants, and, second, to compare the in vivo lung deposition of radiolabeled salbutamol from these three spacer devices in an intubated, spontaneously breathing rabbit model.

\section{METHODS}

\section{In Vivo Study}

Lung deposition of radiolabeled Ventoline (chlorofluorocarbon salbutamol, GlaxoSmithKline) pressurized metered-dose inhaler administered via the three spacer devices was measured in an animal "infant" model. Six healthy male New-Zealand White rabbits, mean weight $2.5-4 \mathrm{~kg}$, were studied. Each rabbit was tested with all three spacer devices. The order in which the spacer devices were used for each rabbit was randomized. Labeling of salbutamol pressurized metered-dose inhaler was performed using a variation of the technique previously described by Summer (15). Pertechnetate (99m $\mathrm{TcO}_{4}{ }^{-}$) was extracted from saline using methyl ethyl ketone. A metal canister was filled with the solution and the methyl ethyl ketone was evaporated using a stream of nitrogen. A previously frozen $\left(-70^{\circ} \mathrm{C}\right)$ canister of Ventoline was opened and the contents were immediately poured into the metal canister containing the radioactivity. This canister was sealed with a Neotechnic 356 metering valve (GlaxoSmithKline) using a Socage Aerosol Crimper, Model 721.11 (BLM, Greenwich, CT, U.S.A.), and placed into an ultrasonic bath for $5 \mathrm{~min}$. The labeled pressurized metered-dose inhaler was placed in a horizontal shaker for $30 \mathrm{~min}$ and allowed to reach room temperature before calibration and use. The total weight was also obtained to ensure that no leakage of propellant occurred over the time of the experiment. Twenty doses of radioactive labeled salbutamol were released onto individual cotton wool balls and each was assayed for radioactivity. The first five doses were priming doses and discarded. The mean of the 15 subsequent doses determined the mean administered dose, in microcuries. Only canisters providing consistent puffs with $<10 \%$ variation in activity were used in study.

Three puffs of radiolabeled Ventoline were delivered at 1-min intervals to spontaneously breathing, lightly anesthetized, intubated rabbits. Anesthesia was administered initially with an intramuscular injection of xylazine $(5 \mathrm{mg} / \mathrm{kg})$ followed, $10 \mathrm{~min}$ later, by ketamine $(55 \mathrm{mg} / \mathrm{kg})$ and acepromazine maleate $(0.75 \mathrm{mg} / \mathrm{kg})$. Heart rate and oxygen saturation were monitored by pulsed oximetry (Ohmeda Model, Ohmeda Biox, Louisville, CO, U.S.A.). Rabbits were intubated with a Portex 3.0-mm endotracheal tube (SIMS Portex Ltd., Hythe, Kent, U.K.) to $13 \mathrm{~cm}$ at the teeth. They were ventilated with continuous positive airway pressure (Servo Ventilator 300, Siemens Elema AB, Solna, Sweden), with warm humidified air after intubation to maintain a pulsed oximetry of approximately $95 \%$. When their tidal volumes reached $15-20 \mathrm{~mL}$, they were disconnected from the ventilator and allowed to breathe room air during the dosing and imaging, further mimicking the conditions of a spontaneously breathing infant. The spacer devices were then connected to the endotracheal tube with connectors that allowed proper use of the valves and minimized the introduction of dead space (modified face mask allowing expiratory flow for Aerochamber-Infant, putty for Babyhaler, and small 3-mL volume plastic adaptor for NESspacer). None of the spacer devices were fitted with a filter to collect the exhaled aerosol. Before delivery, each pressurized metered-dose inhaler was shaken and primed with three puffs. Delivery of the three subsequent puffs was carried out with the animals lying in their left side (Fig. 1). Doses were administered when the rabbits were seen to be breathing and could move the valves of the spacer device. Immediately after the three doses, the rabbits were scanned for $2 \mathrm{~min}$ in the supine position using a Ohio Nuclear large field-of-view gamma camera (Model 410, Ohio Nuclear Solon, OH, U.S.A.) connected to a personal computer. The amount of radiolabeled salbutamol deposited in the spacer device, endotracheal tube, trachea, lungs, and body was determined from the digitized images and by applying attenuation coefficients. The total body outline and regions of interest outlining the trachea and lungs were drawn on the digitized images and the counts recorded in each region determined. The amount of radioactivity remaining in the endotracheal tube and spacer device were determined by first imaging them separately on the gamma camera, drawing regions of interest and determining the counts in each region, and then later by counting these items in a dose calibrator (Capintec Inc., Pittsburgh, PA, U.S.A.). Corrections for decay of technetium were made on all measurements. At the end of the experiment, the rabbits were extubated and housed in their boxes for a minimum of $2 \mathrm{~d}$ before initiating a new experiment.

The study was approved by the Animal Research Ethics Committee of McMaster University.

\section{In Vitro Study}

Emitted dose of salbutamol. The emitted dose of salbutamol (in micrograms per $100 \mu \mathrm{g}$ salbutamol) was determined by collecting individual doses from Ventoline pressurized metered-dose inhalers (GlaxoSmithKline; $100 \mu \mathrm{g}$ salbutamol per actuation) alone and then via Aerochamber-Infant, Babyhaler, and NES-spacer. The tube for single-dose determination,

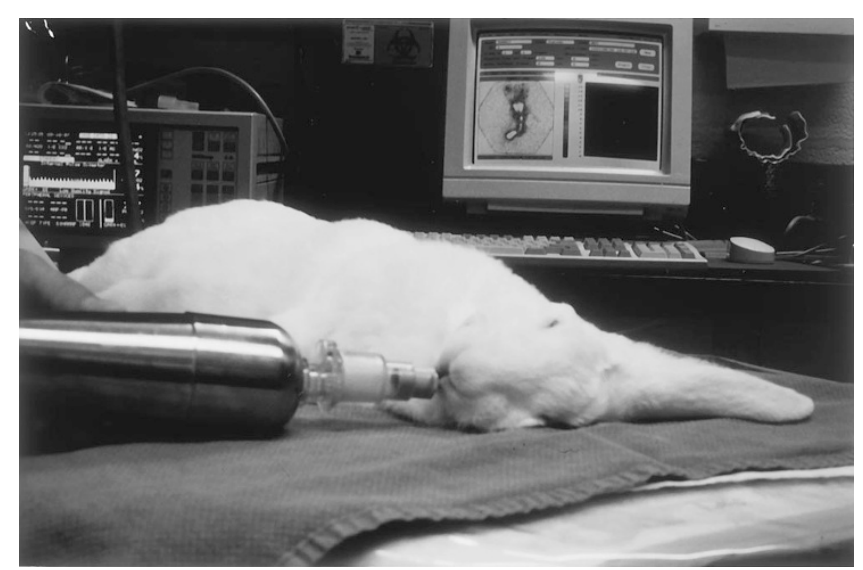

Figure 1. Experimental setup showing the delivery of labeled salbutamol pressurized metered-dose inhaler (Ventoline) from a small-volume spacer device in lightly anesthetized and intubated (Portex 3.0-mm endotracheal tube) spontaneously breathing New Zealand White male rabbits. 
adapted from the United States Pharmacopeia recommendations, was equipped with a Gelman type A/E filter (Pall Gelman Laboratory, Ann Arbor, MI (U.S.A.)) and operated by drawing the aerosol from the pressurized metered-dose inhaler into the tube at a constant suction flow rate of $28.3 \mathrm{~L} / \mathrm{min}$. Three new pressurized metered-dose inhalers and three sets of new spacer devices were used. Each inhaler was used alone and with one of each of the three spacer devices. Before use and between each experiment, the plastic spacer devices were washed with lukewarm water and allowed to dry in ambient air. Immediately before each experiment, the pressurized metered-dose inhaler was vigorously shaken for $30 \mathrm{~s}$ and primed by firing one actuation. This procedure was repeated twice for a total of three priming actuations. The pressurized metereddose inhaler was then shaken for $30 \mathrm{~s}$ and, with the $28.3 \mathrm{~L} / \mathrm{min}$ suction flow on, actuated directly into the collection tube or into the spacer device connected to the collection tube. The flow was stopped $10 \mathrm{~s}$ after the actuation. The pressurized metered-dose inhaler was removed from the tube and stored on its side. This operation was repeated with a fresh sampling tube collecting 10 doses per pressurized metered-dose inhaler and spacer device, for a total of 30 measurements per group.

After the dose collection, each collection tube was hermetically closed. At the distal end, the filter was pushed into the tube and sealed with a second cap. Ten milliliters of solvent $(0.1 \mathrm{~N}$ sodium hydroxide) solution was introduced into each tube to soak the filter. The tubes, with the filters and the solution, were shaken on an agitator for $30 \mathrm{~min}$. An aliquot was then collected from each tube and filtered with a $0.2 \mu \mathrm{m}$ filter (Nylon Acrodisc, Gelman Sciences Inc., Montreal, Quebec, Canada). The amount of drug was assayed by spectrophotometry (Hitachi Spectrophotometer, Model 100-60, Unicam Instrument Ltd, Cambridge, England) at a wavelength of $243 \mathrm{~nm}$. Measuring conditions were mean temperature $23.9^{\circ} \mathrm{C}$ (range $23-26^{\circ} \mathrm{C}$ ) and mean relative humidity $42.3 \%$ (range $21-63 \%$ ).

Particle size distribution of salbutamol. The particle size distribution was measured using an eight-stage nonviable Anderson cascade impactor with a United States Pharmacopeia stainless steel inlet, operated at $28.3 \mathrm{~L} / \mathrm{min}$. Five puffs of salbutamol administered at 30-s intervals were used for each sizing run. The different parameters [fine particle fraction (percentage of particles $<5.8 \mu \mathrm{m}$ ), mass median aerodynamic diameter $(\mu \mathrm{m})$, and geometric SD] were determined from the cumulative mass distribution plot.

Measurements for the pressurized metered-dose inhaler alone or pressurized metered-dose inhaler plus spacer device were performed twice and three sets of pressurized metereddose inhalers and spacer devices were used for a total of six measurements per group. The stage plates of the impactor were washed with solvent $(0.1 \mathrm{~N}$ sodium hydroxide) and the amount of drug determined by spectrophotometry. The same protocol was observed for washing and drying the spacer devices or actuating the pressurized metered-dose inhalers as outlined previously. The fine particle dose (product of the emitted dose by the fine particle fraction, in micrograms per $100 \mu \mathrm{g}$ salbutamol) was calculated in each case ( $n=6$ per group).

\section{Statistical Analysis}

The variability of the in vitro measurements in each set (pressurized metered-dose inhaler plus spacer device combination) was calculated before pooling the results. The results were expressed as mean \pm SD. Comparison between groups of rabbits were made using 2-tailed unpaired $t$ test and MannWhitney Rank Sum test when appropriate. For comparison of repeated measurements in the same animals, the paired $t$ test or Wilcoxon signed rank test were used. Comparison among multiple groups was made using an one way analysis of variance (ANOVA) and the Student-Newman-Keuls test for pairwise multiple comparisons.

\section{RESULTS}

The in vitro measurements for each group were not statistically different, allowing the results for emitted dose and particle size measurements to be pooled.

The results for emitted dose (micrograms per $100 \mu \mathrm{g}$ salbutamol), fine particle dose (micrograms per $100 \mu \mathrm{g}$ salbutamol), mass median aerodynamic diameter (micrometers) and geometric SD are shown Table 1. The mean emitted dose of salbutamol from the pressurized metered-dose inhaler was always greater than with the pressurized metered-dose inhaler and any of the spacer devices. As expected, all the spacer devices increased the fine particle dose of salbutamol compared with the pressurized metered-dose inhaler alone, with the NES-spacer and Babyhaler having similar delivery despite the lack of optimal antistatic treatment of the Babyhaler. The mass median aerodynamic diameter and the geometric SD were similar for all groups.

A total of six different labeled canisters were used for the in vivo study. The mean dose for all $99 \mathrm{~m} \mathrm{TcO}_{4}{ }^{-}$-salbutamol pressurized metered-dose inhalers was $300.1 \pm 95.7 \mu \mathrm{Ci}$ with the NES-spacer, $306.7 \pm 127.8 \mu \mathrm{Ci}$ with the Babyhaler, and

Table 1. In vitro characteristics of salbutamol delivered from a pressurized metered-dose inhaler (Ventoline) and three small-volume spacer devices

\begin{tabular}{lrrrr}
\hline & Ventoline & Aerochamber-Infant & Babyhaler & NES-spacer \\
\hline Emitted dose* $(\mu \mathrm{g} / 100 \mu \mathrm{g})$ & $96.9 \pm 6.9 \ddagger$ & $53.7 \pm 5.1 \S$ & $65.0 \pm 4.4 \S$ & $73.0 \pm 5.4 \S$ \\
Fine particle dose $\dagger(\mu \mathrm{g} / 100 \mu \mathrm{g})$ & $40.3 \pm 8.4 \ddagger$ & $52.4 \pm 5.1 \S$ & $64.4 \pm 4.3 \S$ & $66.4 \pm 5.8 \S$ \\
Mass median aerodynamic diameter $(\mu \mathrm{m})$ & $2.6 \pm 0.08$ & $2.7 \pm 0.05$ & $2.7 \pm 0.05$ & $2.7 \pm 0.02$ \\
Geometric SD & $1.5 \pm 0.08$ & $1.5 \pm 0.02$ & $1.5 \pm 0.03$ & $1.5 \pm 0.01$ \\
\hline
\end{tabular}

Results are expressed as mean \pm SD and compared by ANOVA.

* Ventoline $>$ NES-spacer $>$ Babyhaler $>$ Aerochamber-Infant $(p<0.05)$.

$\dagger$ NES-spacer $=$ Babyhaler $>$ Aerochamber-Infant $>$ Ventoline $(p<0.05)$.

$†$ Ex-actuator

$\S$ Ex-spacer device 
$267.3 \pm 107.1$ with the Aerochamber-Infant. The efficiency of our radiolabeling procedure was high, between $80 \%$ and $95 \%$. The six rabbits were all able to complete the study, i.e. three experiments (one spacer device tested per experiment) per rabbit were made, without any adverse incident or death. Respiratory rate varied between rabbits from 25 to 48 breaths per minute. As expected, heart rate increased with dosing (from $186.4 \pm 20.2$ to $209.8 \pm 19.6$ beats per minute). Pulsed oximetry was normal throughout the experiments. An example of the images obtained with the three spacer devices for one rabbit is shown Figure 2.

Lung deposition (Table 2), expressed as a percentage of the radioactive emitted dose (pressurized metered-dose inhaler dose ex-actuator), was significantly different between the groups $(p=0.002)$. After administration via a $3.0-\mathrm{mm}$ endotracheal tube, the mean lung deposition was $3.53 \pm 1.55 \%$ for NES-spacer, $2.14 \pm 0.64 \%$ for Aerochamber-Infant, and 0.36 $\pm 0.34 \%$ for Babyhaler. Greater variability in lung deposition was found for Babyhaler (coefficient of variation $=110.7 \%$ for the Babyhaler, $44 \%$ for the NES-spacer, and $29.5 \%$ for the Aerochamber-Infant; $p<0.05$ ). However, the main comparison between the spacer devices was based on both lung and body deposition. Indeed, the body dose, as a percentage of the emitted dose from the pressurized metered-dose inhaler, consisted of radioactivity absorbed from the lung over the 3-min pressurized metered-dose inhaler administration time and 2-min imaging. NES-spacer induced the greatest systemic absorption (1.87\%) compared with the Aerochamber-Infant $(0.77 \%)$ and Babyhaler $(0.16 \% ; p<0.05)$. Including the body deposition, the metallic NES-spacer delivered significantly more salbutamol to the intubated rabbits than the two other spacer devices $(5.40 \pm 2.40 \%$ versus $2.91 \pm 0.86 \%$ for Aerochamber-Infant and $0.52 \pm 0.46 \%$ for Babyhaler; $p=$ 0.002).

Deposition of radiolabeled salbutamol was maximal in the spacer devices (between $39 \%$ and $71 \%$ of the radioactive emitted dose according to the spacer device used) and was lowest in the metallic spacer device compared with the plastic spacer devices $(p=0.001)$. Thus, at the mouthpiece of the spacer devices, $29 \%$ of the radioactive emitted dose was available from the Aerochamber-Infant compared with 43\% from the Babyhaler and nearly $60 \%$ from the NES-spacer. However, a significant portion of drug was lost, probably in the exhaled air, as only $48.5 \%$ of the radioactive emitted dose was measured in the spacer device, the endotracheal tube, the trachea, the lungs, or the body of the rabbits for the NESspacer, $58.7 \%$ for the Babyhaler, and $75.8 \%$ for the Aerochamber-Infant (Table 2). Expressing the dose to the rabbit lungs plus body as a percentage of this mean value for the emitted ex-spacer device dose, rather than the emitted ex-actuator dose into the spacer devices, the mean amount deposited from the Aerochamber-Infant was calculated as $10.2 \%, 1.2 \%$ for the Babyhaler, and $8.9 \%$ for the NES-spacer.

\section{DISCUSSION}

Our results have shown that the lung and body deposition of radiolabeled salbutamol in intubated, spontaneously breathing rabbits is greater with the NES-spacer than with the Aerochamber-Infant and the Babyhaler. Some discrepancies were noted between the in vitro and in vivo data measuring delivery of salbutamol via small valved spacer devices. Assessed by the fine particle dose at a constant flow of $28.3 \mathrm{~L} / \mathrm{min}$, in vitro salbutamol delivery was greater with the NES-spacer and Babyhaler than with the Aerochamber-Infant. However, in vivo deposition measured in the rabbits from the Babyhaler was significantly lower than with the other spacer devices.

In vitro studies describing the delivery of salbutamol via spacer devices have been reported by many authors. However, studies using small-valved spacer devices are less frequent and conducted under experimental conditions different from ours, with the use of different constant inspiratory flows $(10,30$, or $60 \mathrm{~L} / \mathrm{min}$ ) or the use of simulated breathing patterns, that would more accurately reflect the drug deposition into the lungs, or the use of reduced static spacers $(8,16-19)$. All experiments have usually demonstrated that the NES-spacer

\section{NES Aerochamber Babyhaler}

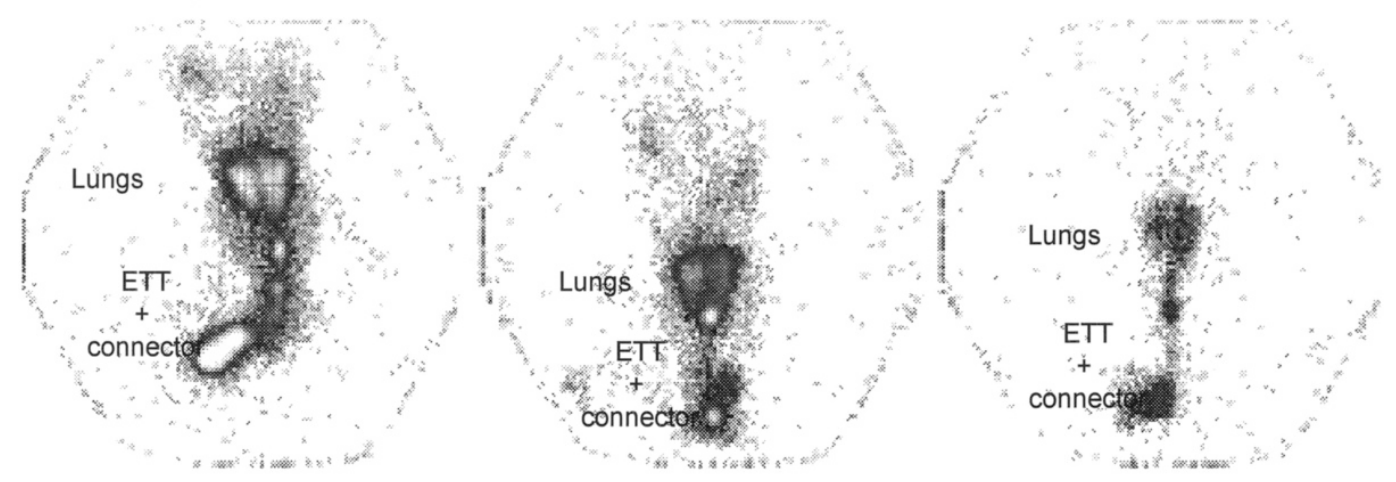

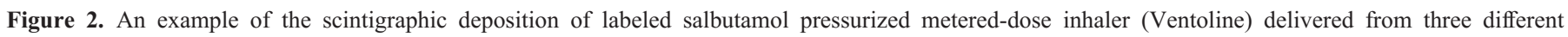

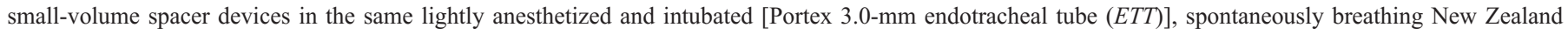

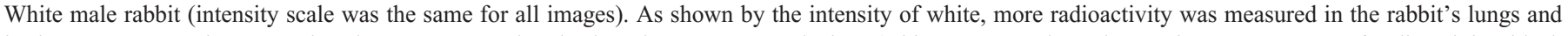

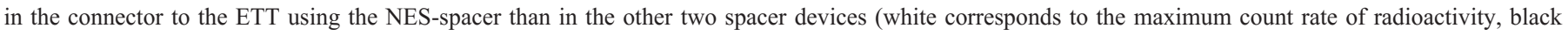
to the minimum). 
Table 2. Deposition of radiolabeled salbutamol pressurized metered-dose inhaler (Ventoline) delivered through small-volume spacer devices in lightly anesthetized and intubated (Portex $3.0 \mathrm{~mm}$ ), spontaneously breathing New Zealand White male rabbits. Deposition equals percentage emitted (ex-actuator) dose (mean $\pm S D)$.

\begin{tabular}{lccc}
\hline & $\begin{array}{c}\text { Aerochamber-Infant } \\
(n=6)^{*}\end{array}$ & $\begin{array}{c}\text { Babyhaler } \\
(n=6)^{*}\end{array}$ & $\begin{array}{c}\text { NES-spacer } \\
(n=6)^{*}\end{array}$ \\
\hline Spacer devicet & $71.5 \pm 13.4$ & $57.4 \pm 7.80$ & $39.1 \pm 13.3$ \\
Endotracheal tube & $1.34 \pm 0.76$ & $0.75 \pm 0.99$ & $3.91 \pm 1.39$ \\
Trachea & $0.00 \pm 0.00$ & $0.00 \pm 0.01$ & $0.10 \pm 0.25$ \\
Lung & $2.14 \pm 0.64$ & $0.36 \pm 0.34$ & $3.53 \pm 1.55$ \\
Lung + body & $2.91 \pm 0.86$ & $0.52 \pm 0.46$ & $5.40 \pm 2.40$ \\
\hline
\end{tabular}

$* n=6$ rabbits, randomized, cross-over, with all three spacer devices.

$\dagger$ Student-Newman-Keuls $t$ test.

$\ddagger$ Measured in the spacer devices.

delivered more salbutamol than all small plastic volume spacer devices, even from a reduced static Babyhaler or Aerochamber-Infant $(2,8)$. We did not measure the electrostatic charges on the spacer devices nor did we use a full antistatic treatment for spacer devices, that is, immersion in diluted ionic detergent or use of an antistatic lining or an antistatic paint $(8,16-19)$. We washed the spacer devices with lukewarm water and allowed them to dry at room air. Clark (20) demonstrated that washing a plastic spacer device (Volumatic, GlaxoSmithKline) in water was as effective as an antistatic paint in reducing the effects of static charge on salbutamol delivery, but other authors $(8,17-19)$ have shown that washing in water alone had a variable and unpredictable effect on spacer electrostatic charge. That in vivo effects of static charge may not support the in vitro results has been further demonstrated in a recent crossover study in 90 young children with asthma (21). The change in peak expiratory flows measured after inhalation of hydrofluoroalkane salbutamol from the NES-spacer compared with static plastic spacer devices or the same plastic spacer devices treated to eliminate static charge were the same. In our study, the fine particle dose of salbutamol, measured in vitro, was similar when administered via NES-spacer or Babyhaler. In vitro, the Aerochamber-Infant resulted in a slightly lower fine particle dose of salbutamol than the two other spacer devices but was always greater compared with the pressurized metered-dose inhaler alone.

A number of studies have shown that it is possible to deliver salbutamol with a pressurized metered-dose inhaler through a small spacer device or by nebulization to nonintubated spontaneously breathing infants. Most of these studies were pharmacodynamic studies with an assessment of the bronchodilator response in asthmatic or BPD patients by the changes in pulmonary function tests $(1,3,7,22-25)$. In one study, the lung deposition of salbutamol was indirectly measured from the amount of drug deposited on a filter placed between the inhalation system and the patient (26). However, this methodology usually overestimates the lung deposition because of lack of branching airways as in normal lung and the retention of the smallest particles normally exhaled onto the filter. To our knowledge, only two scintigraphic studies, in a total of 28 patients, have estimated pressurized metered-dose inhaler lung deposition in young children or infants with asthma, cystic fibrosis, or BPD $(6,7)$. In these studies, from 0.12 to $2.26 \%$ of the nominal dose of radiolabeled salbutamol was shown to be delivered through the Aerochamber-Infant used with a face mask. This lower delivery compared with that for adults prompted Tal (6) to suggest giving young children the same therapeutic doses as recommended for older patients.

Animal lung models have significant limitations as surrogate models for infants. The airways of animals have a branching pattern different from that in humans and the aerosol deposition in healthy animal lungs may be different from that in the diseased lungs of real patients. Despite these limitations and to avoid exposure of infants to radioactivity, we have carefully chosen this method to simulate, as closely as possible, drug inhalation from spacer devices in spontaneously breathing infants or neonates. Our results in intubated but spontaneously breathing rabbits with normal lungs confirm that delivery of salbutamol to small lungs is low. The lung deposition observed in this study for the Aerochamber-Infant is similar to those previously reported in human in vivo scintigraphic studies (6, 7). Deposition was statistically increased when using the NESspacer rather than the two other spacer devices, with a mean lung and body deposition of $5.40 \pm 2.40 \%$. The mean dose of radioactive salbutamol was nearly $60 \%$ of the actuated dose at the output of the NES-spacer, $42.6 \%$ for the Babyhaler, and $28.5 \%$ for the Aerochamber-Infant. The large differences between the amount of salbutamol available for inhalation and the amount of salbutamol actually deposited in the lungs may likely be explained by the low tidal volume of our rabbits (27). The $15-\mathrm{mL}$ tidal volume of our rabbits, assuming human tidal volume/body weight ratio of at least $5 \mathrm{~mL} / \mathrm{kg}$, would be comparable to a $3-\mathrm{kg}$ baby at most. With the $15-\mathrm{mL}$ tidal volume of the rabbits, the valves of Babyhaler were difficult to open. In addition, the larger dead space of Babyhaler relative to Aerochamber-Infant and NES-spacer may have favored deposition of the drug in the Babyhaler.

In conclusion, our study demonstrates that the delivery of salbutamol aerosol is low via small valved spacer devices in spontaneous breathing rabbits intubated with 3.0-mm endotracheal tube. Our reported values for lung deposition are similar to the known lung deposition in infants with pulmonary disease using the Aerochamber-Infant and shows a greater delivery of drug, both in vitro and in vivo, for the NES-spacer. From in vitro measurements, the Babyhaler is as efficient as the NESspacer, but the combination of low tidal volume and large dead space, does not allow optimal delivery in vivo. Further in vivo deposition studies in a variety of other clinical settings should be performed but, from our results, either the AerochamberInfant or the metallic $250-\mathrm{mL}$ valved spacer devices can be 
used to deliver pressurized aerosols successfully to spontaneously breathing young infants.

\section{REFERENCES}

1. Kraemer R, Birrer P, Modelska K, Aebischer CC, Schöni MH 1992 A new babyspacer device for aerosolized bronchodilator administration in infants with bronchopulmonary dysplasia. Eur J Pediatr 151:57-60

2. Bisgaard H, Anhoj J, Klug B, Berg E 1995 A non-electrostatic spacer for aerosol delivery. Arch Dis Child 73:226-230

3. Gappa M, Gärtner M, Poets CF, Von der Hardt H 1997 Effects of salbutamol delivery from a metered-dose inhaler versus jet nebulizer on dynamic lung mechanics in very preterm infants with chronic lung disease. Pediatr Pulmonol 23:442-448

4. Torres Jr A, Anders M, Anderson P, Heulitt MJ 1997 Efficacy of metered-dose inhaler administration of albuterol in intubated infants. Chest 112:484-490

5. Everard ML 1994 Studies using radiolabeled aerosols in children. Thorax 49:1259_ 1266

6. Tal A, Golan H, Grauer N, Aviram M, Albin D, Quastel MR 1996 Deposition pattern of radiolabeled salbutamol inhaled from a metered-dose inhaler by means of a spacer with mask in young children with airway obstruction. J Pediatr 128:479-484

7. Fok T, Monkman S, Dolovich M, Gray S, Coates G, Paes B, Rashid F, Newhouse M, Kirpalani H 1996 Efficiency of aerosol medication delivery from a metered-dose inhaler versus jet nebulizer in infants with bronchopulmonary dysplasia. Pediatr Pulmonol 21:301-309

8. Wildhaber JH, Devadason SG, Eber E, Hayden MJ, Everad ML, Summers QA LeSouëf PN 1996 Effect of electrostatic charge, flow, delay and multiple actuations on the in vitro delivery of salbutamol from different small volume spacers for infants. Thorax 51:985-988

9. Barry PW, O'Callaghan C 1997 The output of salbutamol from spacer devices. Am J Respir Crit Care Med 155:A671

10. Wildhaber JH, Hayden MJ, Dore ND, Devadason SG, LeSouëf PN 1998 Salbutamol delivery from hydrofluoroalkane pressurized metered-dose inhaler in pediatric ventilator circuits: an in vitro study. Chest 113:186-191

11. Everard ML, Clark AR, Milner AD 1992 Drug delivery from holding chambers with attached facemask. Arch Dis Child 67:580-585

12. Coleman DM, Kelly HW, McWilliams BC 1996 Determinants of aerosolized albuterol delivery to mechanically ventilated infants. Chest 109:1607-1613

13. O'Callaghan C, Hardy J, Stammers J, Stephenson TJ, Hull D 1992 Evaluation of techniques for delivery of steroids to lungs of neonates using a rabbit model. Arch Dis Child 67:20-24
14. Fok TF, Al-Essa M, Monkman S, Dolovich M, Girard L, Coates G, Kirpilani H 1997 Pulmonary deposition of salbutamol aerosol delivered by metered dose inhaler, jet nebulizer, and ultrasonic nebulizer in mechanically ventilated rabbits. Pediatr Res 42:721-727

15. Summer QA, Hollingsworth A, Clark AR, Fleming J, Holgate ST 1990 The preparation of radiolabelled aerosol of nedocromil sodium for administration by metereddose inhaler that accurately preserves particle size distribution of the drug. Drug Invest 2:90-98

16. O'Callaghan C, Lynch J, Cant M, Robertson C 1993 Improvement in drug delivery from spacer devices by use of an antistatic lining. Thorax 48:603-606

17. Barry PW, O'Callaghan C 1996 Inhalational drug delivery from seven different spacer devices. Thorax 51:835-840

18. Wildhaber JH, Devadason SG, Hayden MJ, James R, Dufty AP, Fox RA, Summers QA, LeSouëf PN 1996 Electrostatic charge on a plastic spacer device influences the delivery of salbutamol. Eur Respir J 9:1943-1946

19. Barry PW, O'Callaghan C 1997 In vitro comparison of the amount of salbutamol available for inhalation from different spacer devices. Eur Respir J 10:1345-1348

20. Clark DJ, Lipworth BJ 1996 Effect of multiple actuations, delayed inhalation and antistatic treatment on the lung bioavailability of salbutamol via a spacer device. Thorax 51:981-984

21. Dompeling E, Oudesluys-Murphy AM, Janssens HM, Hop W, Brinkman JG, Sukhai RN, de Jongste JC 2001 Randomised controlled study of clinical efficacy of spacer therapy in asthma with regard to electrostatic charge. Arch Dis Child 84:178-182

22. Conner WT, Dolovich MB, Frame RA, Newhouse MT 1989 Reliable salbutamol administration in 6- to 36-month-old children by means of metered-dose inhaler and aerochamber with mask. Pediatr Pulmonol 6:263-267

23. Kraemer R, Frey U, Sommer CW, Russi E 1991 Short-term effect of albuterol delivered via a new auxiliary device in wheezy infants. Am Rev Respir Dis 144:347351

24. Clarke JR, Aston H, Silverman M 1993 Delivery of salbutamol by metered-dose inhaler and valved spacer to wheezy infants: effect on bronchial responsiveness. Arch Dis Child 69:125-129

25. Fok TF, Lam K, Chan CK, Ng PC, Zhuang H, Wong W, Cheung KL 1997 Aerosol delivery to non-ventilated infants by metered-dose inhaler: should a valved spacer be used? Pediatr Pulmonol 24:204-212

26. Wildhaber JH, Devadason SG, Hayden MJ, Eber E, Summers QA, LeSouëf PN 1997 Aerosol delivery to wheezy infants: a comparison between a nebulizer and two small volume spacers. Pediatr Pulmonol 23:212-216

27. Dolovich M 1999 Aerosol delivery to children: what to use, how to choose. Pediatr Pulmonol Suppl 18:79-82 